\title{
A Dexmedetomidina para Sedação, por Via Venosa, não Interfere com a Duração dos Bloqueios Sensitivo e Motor da Raquianestesia *
}

\author{
Intravenous Dexmedetomidine for Sedation does not Interfere with \\ Sensory and Motor Block Duration during Spinal Anesthesia \\ Edno Magalhães, TSA ${ }^{1}$; Luís Cláudio de Araújo Ladeira, TSA ${ }^{2}$; Cátia Sousa Govêia, TSA ${ }^{2}$; Beatriz Vieira Espíndola ${ }^{3}$
}

\section{RESUMO}

Magalhães E, Ladeira LCA, Govêia CS, Espíndola BV - A Dexmedetomidina para Sedação, por Via Venosa, não Interfere com a Duração dos Bloqueios Sensitivo e Motor da Raquianestesia

JUSTIFICATIVA E OBJETIVOS: A anestesia locorregional é uma prática freqüente e de grande aplicabilidade em Anestesiologia. Contudo, o paciente pode tornar-se ansioso, fazendo-se necessária a sedação. Os agentes benzodiazepínicos, opióides e o propofol são amplamente utilizados com este objetivo. Os agonistas alfa ${ }_{2}$-adrenérgicos possuem propriedades hipnóticas e sedativas e são uma alternativa no arsenal terapêutico, conferindo estabilidade hemodinâmica e mínima depressão respiratória. O objetivo deste estudo foi avaliar a segurança e a interferência do uso da dexmedetomidina ou do midazolam, por via venosa, na duração dos bloqueios motor e sensitivo em raquianestesia.

MÉTODO: Foram estudadas 35 pacientes adultas, do sexo feminino, estado físico ASA I e II, submetidas à raquianestesia com bupivacaína a 0,5\% hiperbárica $(15 \mathrm{mg})$, para cirurgia ginecológica eletiva, distribuídas de modo aleatório em dois grupos: grupo $M(n=17)$ - sedação com midazolam em infusão contínua a $0,25 \mu \mathrm{g} \cdot \mathrm{kg}^{-1} \cdot \mathrm{min}^{-1}$ e grupo $D(n=18)$ - sedação com dexmedetomidina em infusão contínua a $0,5 \mu \mathrm{g} \cdot \mathrm{kg}^{-1} \cdot \mathrm{min}^{-1}$. A velocidade de infusão foi ajustada para manter o valor de BIS entre 60 e 80 . Foram analisados os valores de PAS, PAD, FC, $\mathrm{SpO}_{2}, \mathrm{BIS}$, extensão e duração dos bloqueios sensitivo motor (escala de Bromage).

RESULTADOS: Não houve diferença estatística significativa entre os grupos quanto à idade, peso, nível de bloqueio sensitivo, variação na pressão arterial e freqüência cardíaca e na duração dos bloqueios sensitivo e motor.

\footnotetext{
${ }^{*}$ Recebido do (Received from) Hospital Universitário de Brasilia - Universidade de Brasília, Brasilia, DF

1. Professor Adjunto da Área de Clínica Cirúrgica da Faculdade de Medicina da Universidade de Brasília (UnB); Responsável pelo CET da Clínica de Anestesiologia da Universidade de Brasília

2. Médico Assistente da Clínica de Anestesiologia do Hospital Universitário de Brasília - Universidade de Brasília (UnB); Co-responsável pelo CET da Clínica de Anestesiologia da Universidade de Brasília

3. Médica Assistente da Clínica de Anestesiologia do Hospital Universitário de Brasília - Universidade de Brasília (UnB)
}

Apresentado (Submitted) em 04 de julho de 2005

Aceito (Accepted) para publicação em 10 de outubro de 2005

Endereço para correspondência (Correspondence to)

Dr. Edno Magalhães

SOS 113, Bloco C/Apt 406

70376-030 Brasilia, DF

E-mail:ednomag@brturbo.com

(C) Sociedade Brasileira de Anestesiologia, 2006
CONCLUSÕES: A dexmedetomidina utilizada em sedação, por via venosa, não interferiu nos parâmetros hemodinâmicos, duração ou extensão dos bloqueios sensitivo e motor na raquianestesia, representando boa opção para sedação durante anestesia locorregional.

Unitermos: ANALGÉSICOS: dexmedetomidina; ANESTÉSICOS, Local: bupivacaína hiperbárica;TÉCNICAS ANESTÉSICAS, Regional: raquianestesia

\section{SUMMARY}

Magalhães E, Ladeira LCA, Govêia CS, Espíndola BV - Intravenous Dexmedetomidine for Sedation does not Interfere with Sensory and Motor Block Duration during Spinal Anesthesia

BACKGROUND AND OBJECTIVES: The association among local and regional anesthesia is a very useful and common practice. However, some patients may become anxious and require sedation. Benzodiazepines, opioids and propofol are widely used for this aim. Alpha ${ }_{2}$-adrenergic agonists have hypnotic and sedative properties and represent an alternative to promote hemodynamic stability and minor respiratory depression. This study aimed at evaluating the safety and the interference of intravenous dexmedetomidine or midazolam on sensory and motor block duration spinal anesthesia.

METHODS: Thirty five adult female patients, physical status ASA I and II, were submitted to spinal anesthesia with hyperbaric $0.5 \%$ bupivacaine $(15 \mathrm{mg})$ for elective gynecologic surgery. The patients were randomized and distributed in two groups: Group $M(n=17)$ - sedation with $0.25 \mu \mathrm{g} \cdot \mathrm{kg}^{-1} \cdot \mathrm{min}^{-1}$ midazolam continuous infusion and Group $D(n=18)$ sedation with $0.5 \mu \mathrm{g} . \mathrm{kg}^{-1} \cdot \mathrm{min}^{-1}$ dexmedetomidine continuous infusion. Infusion rate was adjusted to maintain BIS between 60 and 80 . The following parameters were evaluated: $S B P, D B P, H R$, $\mathrm{SpO}_{2}, \mathrm{BIS}$ sensory and motor block extension and duration (Bromage scale).

RESULTS: There were no statistically significant differences between groups in age, weight, sensory block level, blood pressure and heart rate variation and sensory and motor block duration.

CONCLUSIONS: Intravenous dexmedetomidine for sedation has not interfered with hemodynamic parameters, spinal anesthesia sensory and motor block duration or extension and it is a good option for sedation during local/regional anesthesia.

Key Words: ANALGESICS: dexmedetomidine; ANESTHETICS, Local: hyperbaric bupivacaine; ANESTHETIC TECHNIQUES, Regional: spinal block

\section{INTRODUÇÃO}

A anestesia locorregional encontra ampla aceitação como método eficaz na moderna prática anestésica. A utilização desta técnica permite, como benefícios, o controle da resposta endócrina e metabólica ao trauma, a redução 
de perda sangüínea durante a cirurgia, a redução da incidência de fenômenos tromboembólicos no pós-operatório e a promoção de analgesia no período pós-operatório ${ }^{1}$.

A anestesia regional, entretanto, pode proporcionar ao paciente algum tipo de desconforto, decorrente da realização do procedimento ou extensão excessiva do período peri-operatório. Torna-se necessário o uso concomitante de medicamentos com propriedades hipnóticas, sedativas e amnésicas. Os benzodiazepínicos, o propofol e os opióides representam fármacos com essas propriedades. A administração dessas drogas proporciona algum conforto aos pacientes. Contudo, são fármacos com efeitos sobre os mecanismos regulatórios da ventilação, podendo levar à depressão da ventilação, hipercarbia e hipoxemia.

Uma alternativa promissora ao uso desses fármacos em

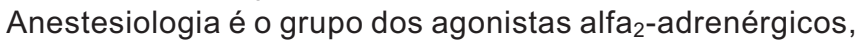
fármacos com excelentes propriedades sedativas e analgésicas associados à ausência de depressão respiratória. A dexmedetomidina é um alfa ${ }_{2}$-agonista com grande seletividade ao receptor específico e tem sido usada como medicação pré-anestésica, como adjuvante à anestesia geral, como sedativo e como medicação pós-operatória ${ }^{2-4}$.

A associação da dexmedetomidina como adjuvante em anestesia locorregional proporcionaria uma interação farmacológica sinérgica com aumento da duração de ação dos anestésicos locais, estabilidade hemodinâmica, sedação, analgesia e ausência de depressão ventilatória ${ }^{2,4}$.

O objetivo do presente estudo foi comparar os parâmetros fisiológicos da sedação por via venosa com midazolam e dexmedetomidina e avaliar a influência da dexmedetomidina, em doses clinicamente indicadas para sedação, sobre a duração dos bloqueios motor e sensitivo promovido na raquianestesia pela bupivacaína hiperbárica.

\section{MÉTODO}

Após a aprovação pelo Comitê de Ética em Pesquisa da Universidade de Brasília foram estudadas 35 pacientes adultas, do sexo feminino, estado físico ASA I ell, submetidas a raquianestesia e sedação por via venosa com dexmedetomidina ou midazolam em infusão contínua para cirurgia ginecológica eletiva, após consentimento prévio e informado. Foram excluídas pacientes com distúrbios reconhecidos de função renal, hepática e cardíaca, portadora de obesidade mórbida, em uso de medicação anti-hipertensiva ou com contra-indicação à anestesia regional.

A medicação pré-anestésica utilizada foi diazepam (10 mg) na véspera e na manhã do dia da cirurgia. Amonitorização incluiu eletrocardiografia contínua (ECGc), pressão arterial não-invasiva (PANI), saturação periférica de oxigênio $\left(\mathrm{SpO}_{2}\right)$ e análise do eletroencefalograma pelo índice bispectral (BIS).

As pacientes foram distribuídas de modo aleatório em dois grupos. O grupo $M(n=17)$ - pacientes submetidas à sedação com midazolam - recebeu infusão por via venosa contínua de midazolam com velocidade inicial de $0,25 \mu \mathrm{g} \cdot \mathrm{kg}^{-1} \cdot \mathrm{min}^{-1}$, ajustada de acordo com o valor de BIS (valores mantidos en- tre 60 e 80). O grupo $D(n=18)$ - pacientes submetidas à sedação com dexmedetomidina - recebeu infusão por via venosa contínua de dexmedetomidina com velocidade inicial de infusão de $0,5 \mu \mathrm{g} \cdot \mathrm{kg}^{-1} \cdot \mathrm{min}^{-1}$, ajustada de acordo com o valor de $\mathrm{BIS}$ (valores mantidos entre 60 e 80 ). O regime de infusão foi ajustado em ambos os grupos visando limitar a hipnose leve ou superficial caracterizando sedação (valores de BIS mantidos entre 60 e 80). Considerando-se a diferença facilmente identificável entre as velocidades de infusão para dexmedetomidina e midazolam, visando o mesmo nível de BIS, optou-se por um estudo não-encoberto.

Após venóclise e monitorização, procederam-se a raquicentese com agulha descartável Quincke $25 \mathrm{G}$ e administração de bupivacaína a $0,5 \%$ hiperbárica $(15 \mathrm{mg})$. Com a paciente em decúbito dorsal horizontal iniciou-se a pesquisa do nível do bloqueio sensitivo (sensibilidade tátil) um minuto após a punção subaracnóidea e em intervalos de cinco minutos posteriormente. A intensidade do bloqueio motor (Bromage) foi avaliada inicialmente até a instalação de bloqueio motor total e, após o término do procedimento cirúrgico, avaliada em intervalos de 10 minutos para a regressão do bloqueio. Iniciou-se a infusão de midazolam ou dexmedetomidina 10 minutos após a realização da anestesia.

Os valores da pressão arterial sistólica (PAS), pressão arterial diastólica (PAD), freqüência cardíaca ( $F C)$, BIS, nível sensitivo e intensidade do bloqueio motor foram avaliados a cada cinco minutos durante o período peri-operatório. Após o término do procedimento cirúrgico, as pacientes foram encaminhadas à sala de recuperação pós-anestésica (SRPA) e observadas no pós-operatório para a pesquisa da regressão dos bloqueios motor e sensitivo. Considerou-se como duração do bloqueio motor o tempo necessário para que a paciente conseguisse fletir o joelho e levantar a perna estendida (ausência de bloqueio motor na escala de Bromage) O tratamento estatístico foi realizado com o teste $t$ de Student e considerou-se como significativo um valor de $p<0,05$.

\section{RESULTADOS}

Os dados demográficos (Tabela I) da população em estudo não apresentaram diferenças estatísticas significativas entre os grupos. Todas as pacientes do estudo apresentaram bloqueio motor completo (impossibilidade de fletir os pés Bromage) e a moda do nível de bloqueio sensitivo foi $\mathrm{T}_{4}$ ( $\mathrm{Ta}$ bela II) em ambos os grupos. As variáveis hemodinâmicas monitorizadas - pressão arterial sistólica, pressão arterial diastólica e freqüência cardíaca (Figuras 1,2e3) não apresentaram diferença estatística significativa entre os grupos nos momentos estudados. O tempo para regressão total de bloqueio motor, utilizando-se a escala de Bromage (Figura 4), foi de $249,7 \pm 31,8 \mathrm{~min}$ no grupo $D$ e de $240,6 \pm 39,2 \mathrm{~min}$ no grupo $M$, não demonstrando diferença estatística significativa $(p=0,455)$ (Tabela III). O tempo para regressão total do bloqueio sensitivo foi de $219,7 \pm 25,4$ min no grupo $D$ e de $197,5 \pm 40,9$ min no grupo $M$, sem demonstrar diferença estatística significativa $(p=0,062)$. 
Tabela I - Dados Demográficos

\begin{tabular}{lcc}
\hline & Grupo D & Grupo M \\
\hline Idade (anos) & $40,82 \pm 5,26$ & $46,17 \pm 7,52$ \\
Peso $(\mathrm{kg})$ & $63,65 \pm 9,55$ & $66,72 \pm 12,07$ \\
\hline
\end{tabular}

Tabela II - Freqüência de Distribuição do Nível de Bloqueio Sensitivo

\begin{tabular}{lcccc}
\hline & $\mathrm{T}_{2}$ & $\mathrm{~T}_{4}$ & $\mathrm{~T}_{6}$ & Total \\
\hline Grupo D & 1 & 15 & 1 & 17 \\
Grupo M & 2 & 15 & 1 & 18 \\
\hline
\end{tabular}

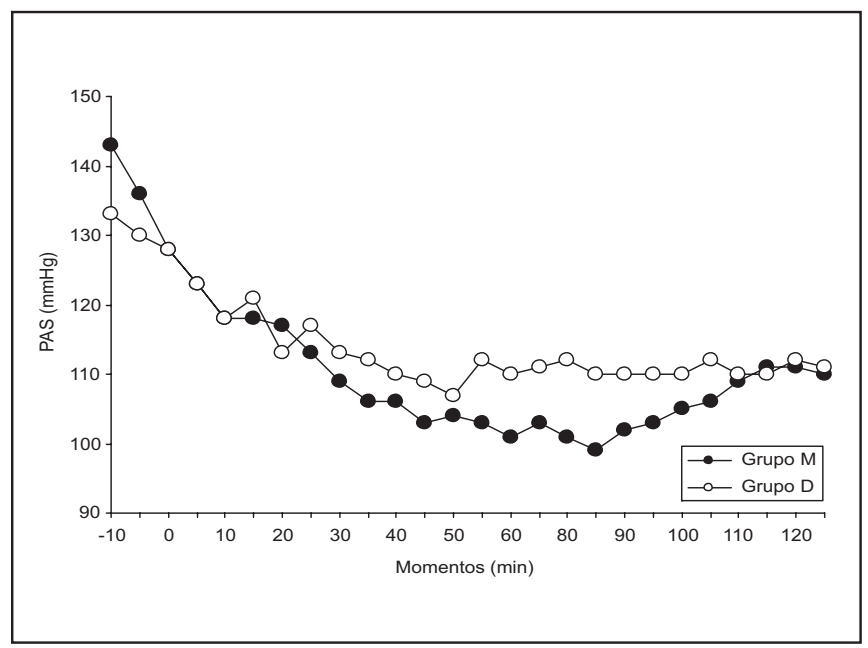

Figura 1 - Variação da Pressão Arterial Sistólica

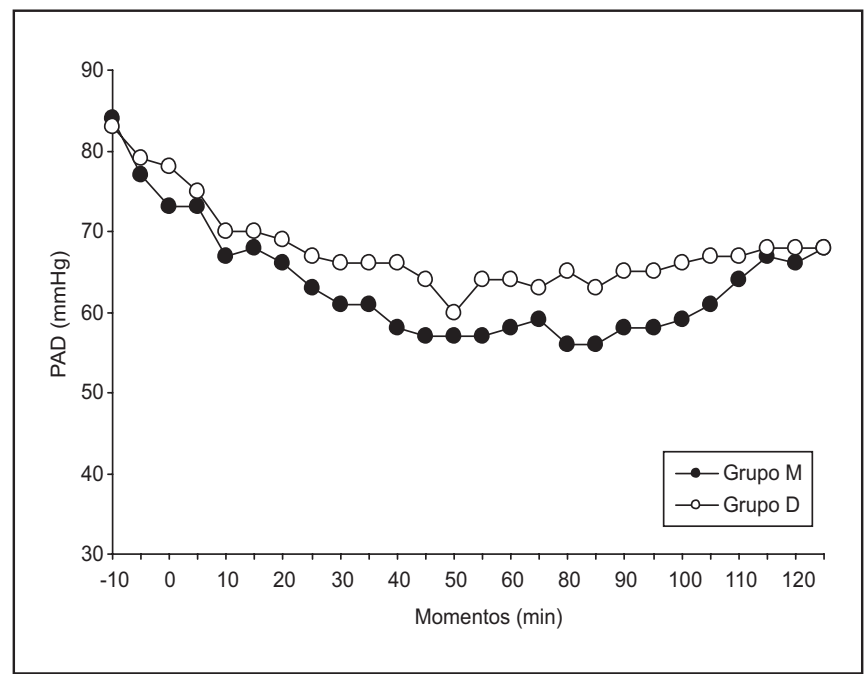

Figura 2 - Variação da Pressão Arterial Diastólica

Revista Brasileira de Anestesiologia

Vol. 56, № 1, Janeiro - Fevereiro, 2006

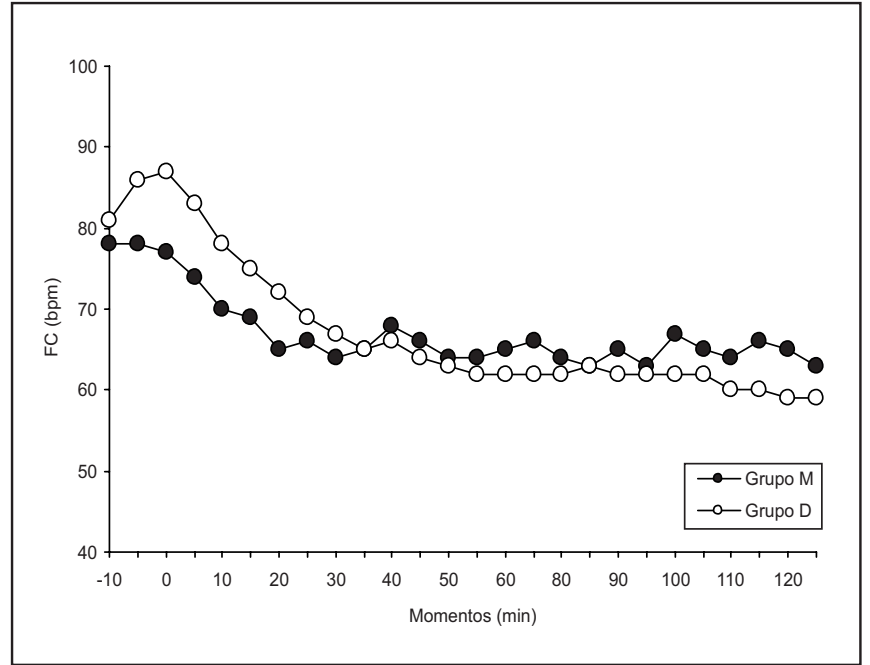

Figura 3 - Variação da Freqüência Cardíaca

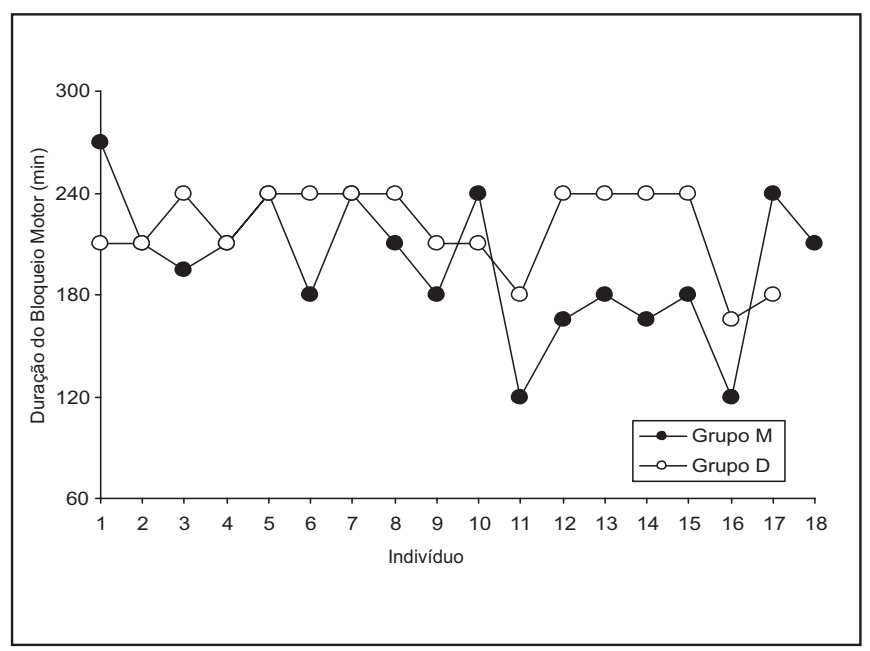

Figura 4 - Distribuição das Pacientes em Relação à Duração do Bloqueio Motor

Tabela III - Duração do Bloqueio Motor

\begin{tabular}{ccc}
\hline & Grupo D & Grupo M \\
\hline Tempo (min) & $249,7 \pm 31,8$ & $240,6 \pm 39,2$ \\
\hline
\end{tabular}

\section{DISCUSSÃo}

Os agonistas dos receptores alfa $_{2}$-adrenérgicos possuem propriedades benéficas, quando utilizados em anestesia. Estes fármacos proporcionam estabilidade hemodinâmica, sedação, redução da necessidade de agentes anestésicos e analgésicos ${ }^{5}$, sem efeitos depressores marcantes da ventilação. O mecanismo de ação destas drogas não se relaciona com alterações da síntese, estocagem ou metabolismo de 
neurotransmissores e é reversível com o uso de agentes vasoativos, agonistas destes receptores ou a interrupção da administração ${ }^{6,7}$. A dexmedetomidina é um agonista com

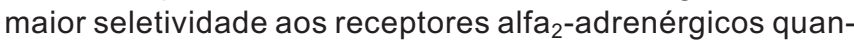
do comparado à clonidina. A dexmedetomidina possui como propriedades farmacocinéticas um rápido início de ação e uma meia-vida curta, proporcionando redução dos níveis plasmáticos de noradrenalina, estabilidade hemodinâmica, sedação e analgesia, sem depressão respiratória ${ }^{8}$.

Alguns mecanismos propostos para a ação dos agonistas alfa $_{2}$-adrenérgicos combinados ao uso de anestésicos locais incluem vasoconstrição, facilitação do bloqueio de fibras $C$ por anestésicos locais, interferência no transporte axonal retrógrado no nível medular e a ação em receptores de nervos periféricos ${ }^{9}$. Alguns relatos na literatura discutem o aumento da duração dos bloqueios sensitivo e motor induzido pelo uso de agentes agonistas alfa ${ }_{2}$-adrenérgicos em bloqueios neuroaxiais $^{9}$

Rhee e col., observaram aumento da duração dos bloqueios sensitivo e motor induzidos pelo uso de clonidina venosa associada à raquianestesia ${ }^{10}$. Memis e col. observaram redução da latência na instalação do bloqueio motor e retardo da recuperação completa da atividade motora quando associou a dexmedetomidina à anestesia venosa regional com lidocaína ${ }^{11}$. A interferência do uso de agentes alfa $_{2}$-adrenérgicos sobre a atividade motora não era o objetivo dos estudos citados. Estudos com o objetivo de caracterizar a real interferência destes fármacos sobre a duração dos bloqueios motor e sensitivo tornam-se necessários e devem ser produzidos.

Autilização de dose única ou repetida em anestesia pode ser uma alternativa inadequada para a mensuração dos efeitos de uma droga. Hu e col., por intermédio de simulações matemáticas, previram o dobro do coeficiente de variação na concentração plasmática de um fármaco, quando administrado por dose única comparada à técnica de infusão contínua ${ }^{12}$. Isto pode acarretar elevadas concentrações plasmáticas de fármacos e a ocorrência de efeitos adversos. O uso de infusão contínua permite a manutenção constante da concentração plasmática de drogas, evitando efeitos reduzidos e a possibilidade de reações adversas.

A utilização de dose única sistêmica em contraponto à infusão contínua pode ser a responsável pelo aumento inesperado da duração do bloqueio motor. Sudo e col. encontraram essa característica em um estudo com animais, no qual a utilização de doses elevadas de dexmedetomidina por via sistêmica possibilitou um aumento da duração do bloqueio motor em animais submetidos à raquianestesia com levobupivacaína, reduzido parcialmente por ioimbina ${ }^{13}$.

Neste estudo não se observou diferença entre a utilização por infusão contínua de dexmedetomidina ou midazolam, nas doses limitadas à produção de sedação, em relação à interferência na duração dos bloqueios sensitivo e motor proporcionados pela raquianestesia com bupivacaína a $0,5 \%$ hiperbárica.

O uso de dexmedetomidina para sedação não mostrou diferença nos valores de pressão arterial e de freqüência cardíaca, quando comparado ao uso de midazolam também para a sedação nesta avaliação. É possível que o uso pré-anestésico do diazepam, um benzodiazepínico de longa meia-vida, tenha influenciado o grau de sedação no período intra-operatório. Entretanto, os dois grupos estudados receberam o mesmo tratamento pré-anestésico e as taxas de infusão do midazolam e da dexmedetomidina foram ajustadas para um valor alvo de BIS. Assim, acredita-se que a redução da necessidade anestésica promovida pelo diazepam tenha sido semelhante para ambos os grupos. Os resultados deste estudo permitem concluir que a dexmedetomidina utilizada em sedação por via venosa, em pacientes submetidas à raquianestesia, representa uma boa opção para sedação, com estabilidade hemodinâmica, proporcionando um nível adequado de hipnose, sem provocar alterações na duração dos bloqueios motor e sensitivo.

\section{Intravenous Dexmedetomidine for Sedation does not Interfere with Sensory and Motor Block Duration during Spinal Anesthesia}

Edno Magalhães, TSA, M.D.; Luís Cláudio de Araújo Ladeira, TSA, M.D.; Cátia Sousa Govêia, TSA, M.D. Beatriz Vieira Espíndola, M.D.

\section{INTRODUCTION}

The combined local/regional anesthesia is widely accepted as an effective method for modern anesthetic practice. This technique allows the control of endocrine and metabolic response to trauma, decreases intraoperative blood loss and the incidence of postoperative thromboembolism, and promotes postoperative analgesia ${ }^{1}$.

Regional anesthesia, however, may promote some type of discomfort caused by the procedure itself or by a prolonged perioperative period, requiring the simultaneous administration of hypnotic, sedative and amnesic drugs. Benzodiazepines, propofol and opioids have these properties and provide some comfort to patients. However, they affect the ventilatory regulatory mechanisms and may lead to respiratory depression, with consequent hypercarbia and hypoxemia.

A promising alternative to these drugs in Anesthesiology is the group of alpha ${ }_{2}$-adrenergic agonists, which have excellent sedative and analgesic properties without respiratory depression. Dexmedetomidine is an alpha ${ }_{2}$-agonist with strong selectivity to the specific receptor and has been used as preanesthetic medication, as adjuvant to general anesthesia, as sedative during trans and postoperative period ${ }^{2-4}$. Dexmedetomidine associated to local/regional anesthesia would provide a synergistic pharmacological interaction prolonging the action of local anesthetics, improving Vol. 56, No 1, Janeiro - Fevereiro, 2006 
hemodynamic stability, sedation and analgesia and not causing respiratory depression ${ }^{2,4}$.

This study aimed at comparing physiological parameters of intravenous sedation with midazolam and dexmedetomidine and at evaluating the influence of dexmedetomidine in doses clinically indicated for sedation, on the duration of sensory and motor block promoted by spinal hyperbaric bupivacaine.

\section{METHODS}

After the Research Ethics Committee, Universidade de Brasília approval and their informed consent, participated in this study 35 adult female patients, physical status ASA I and II, submitted to spinal anesthesia and intravenous sedation with dexmedetomidine or midazolam continuous infusion for elective gynecologic procedures. Exclusion criteria were patients with known renal, liver or heart function disorders, morbidly obese, under anti-hypertensive medication or counterindicated to regional anesthesia.

Patients were premedicated with diazepam $(10 \mathrm{mg})$ the day before and in the morning of the surgery day. Monitoring consisted of continuous ECG, noninvasive blood pressure (NIBP), oxygen peripheral saturation $\left(\mathrm{SpO}_{2}\right)$ and $\mathrm{EEG}$ analysis by bispectral index (BIS).

Patients were randomly distributed in two groups. Group M ( $n$ =17) - sedation with midazolam - intravenous midazolam continuous infusion at initial rate of $0.25 \mu \mathrm{g} \cdot \mathrm{kg}^{-1} \cdot \mathrm{min}^{-1}$ adjusted according to BIS (values maintained between 60 and $80)$. Group $D(n=18)$ - sedation with dexmedetomidine - intravenous dexmedetomidine continuous infusion at initial infusion rate of $0.5 \mu \mathrm{g} \cdot \mathrm{kg}^{-1}$. $\mathrm{min}^{-1}$, adjusted according to BIS (values maintained between 60 and 80 ). Infusion regimen was adjusted in both groups to limit hypnosis to mild or superficial characterizing sedation (BIS between 60 and 80).

Considering the easily identified difference between infusion rates for dexmedetomidine and midazolam aiming at the same BIS level, this study was not blind.

After venous puncture and monitoring, spinal anesthesia was induced with disposable 25G Quincke needle and hyperbaric $0.5 \%$ bupivacaine $(15 \mathrm{mg})$. With patients in the supine position sensory block level started to be investigated (tactile sensitivity) one minute after spinal puncture and then at 5-minute intervals. Motor block intensity (Bromage) was initially evaluated until total motor block installation and then at surgery completion at 10-minute intervals for blockade regression. Midazolam or dexmedetomidine infusion was started 10 minutes after anesthetic induction.

Systolic blood pressure (SBP), diastolic blood pressure (DBP), heart rate (HR), BIS, sensory level and motor block intensity were evaluated every 5 minutes in the perioperative period. At surgery completion patients were referred to the post-anesthetic recovery unit (PACU) and were observed to check motor and sensory block duration. Motor block duration was considered time elapsed before patients were able to bend the knee and raise the stretched leg (no motor block according to Bromage scale).
Student's $t$ test was used for statistical analysis considering significant $p<0.05$.

\section{RESULTS}

Demographics data (Table I) were not statistically different between groups. All patients had total motor block (impossibility of bending feet - Bromage) and sensory block level mode was $\mathrm{T}_{4}$ (Table II) for both groups. Hemodynamic variables - systolic blood pressure, diastolic blood pressure and heart rate (Figures 1, 2 and 3) - were not statistically different between groups in studied moments. Time for total motor block regression according to Bromage scale (Figure 4) was 249.7 \pm 31.8 min for group $D$ and $240.6 \pm 39.2$ min for group $M$, without statistically significant difference $(p=0.455)$ (Table III). Time for total sensory block regression was $219.7 \pm 25.4 \mathrm{~min}$ for group $D$ and $197.5 \pm 40.9$ min fir group $M$, without statistically significant difference $(p=0.062)$.

Table I - Demographics Data

\begin{tabular}{lcc}
\hline & Group D & Group M \\
\hline Age (years) & $40.82 \pm 5.26$ & $46.17 \pm 7.52$ \\
Weight $(\mathrm{kg})$ & $63.65 \pm 9.55$ & $66.72 \pm 12.07$ \\
\hline
\end{tabular}

Table II - Frequency of Sensory Block Level Distribution

\begin{tabular}{lllcc}
\hline & $\mathrm{T}_{2}$ & $\mathrm{~T}_{4}$ & $\mathrm{~T}_{6}$ & Total \\
\hline Group D & 1 & 15 & 1 & 17 \\
Group M & 2 & 15 & 1 & 18 \\
\hline
\end{tabular}

Table III - Motor Block Duration

\begin{tabular}{ccc}
\hline & Group D & Group M \\
\hline Time $(\mathrm{min})$ & $249.7 \pm 31.8$ & $240.6 \pm 39.2$ \\
\hline
\end{tabular}

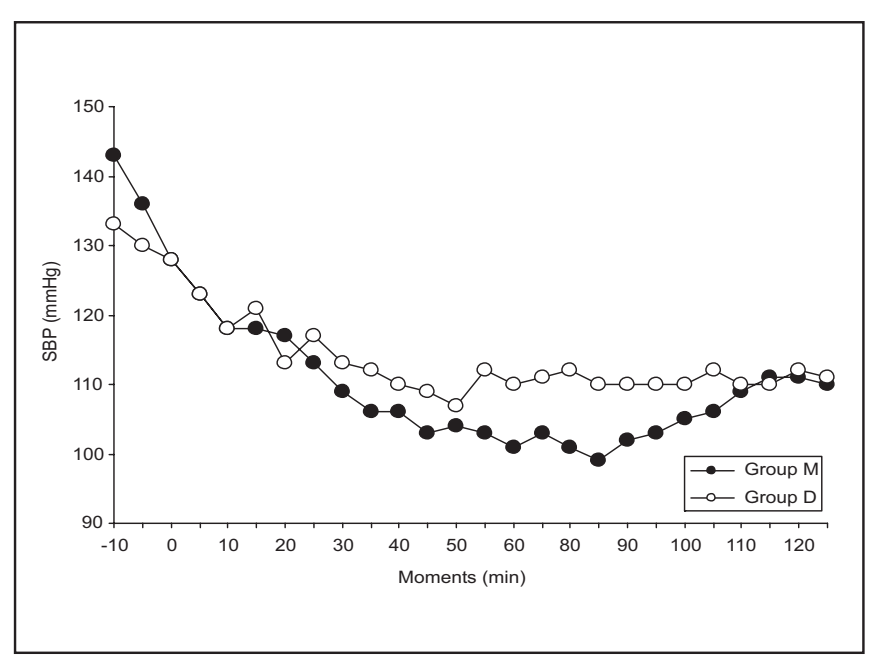

Figure 1 - Systolic Blood Pressure Variation 


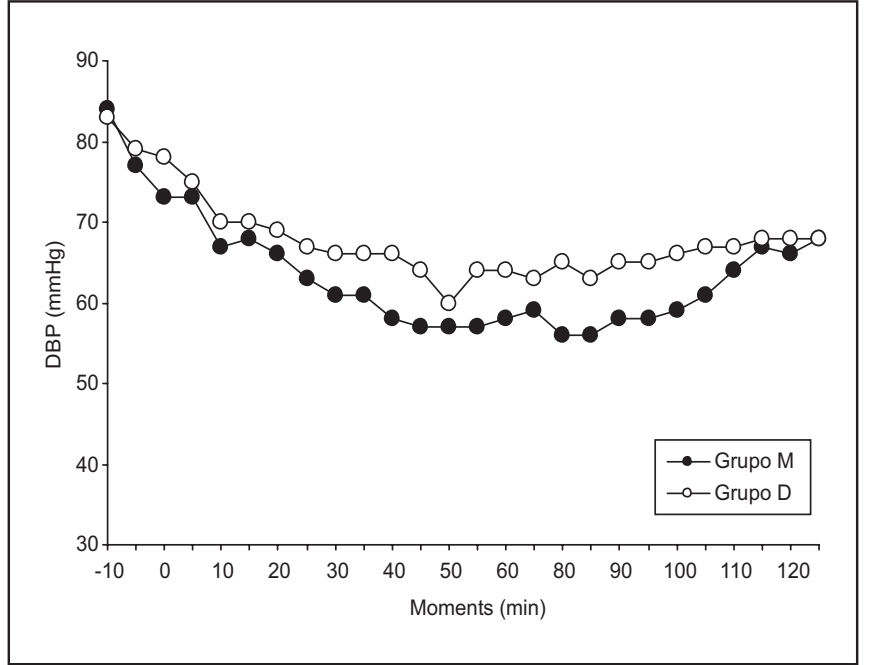

Figure 2 - Diastolic Blood Pressure Variation

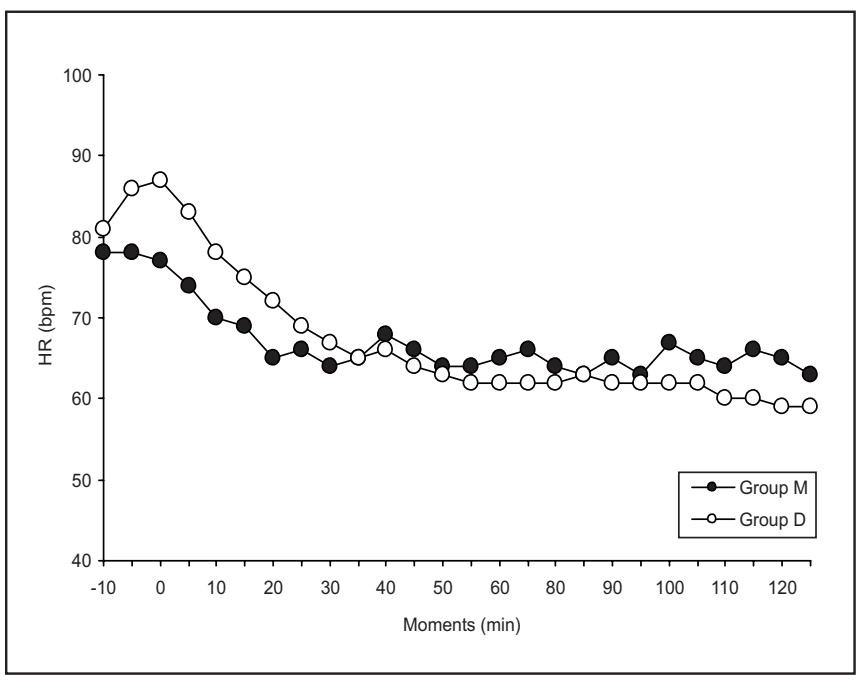

Figure 3 - Heart Rate Variation

\section{DISCUSSION}

Alpha $_{2}$-adrenergic receptor agonists are beneficial for anesthesia. They provide hemodynamic stability and sedation, decrease the need for anesthetics and analgesics ${ }^{5}$, and have no marked depressing effects on ventilation. Their action mechanism is not related to neurotransmitters synthesis, storage or metabolism and is reversible with vasoactive agents, agonists of these receptors, or by withdrawing the drug 6,7 . Dexmedetomidine is more selective to alpha $_{2}$-adrenergic receptors as compared to clonidine. Dexmedetomidine has fast onset and short half-life promoting decreased norepinephrine plasma levels, hemodynamic stability, sedation and analgesia without respiratory depres$\operatorname{sion}^{8}$.

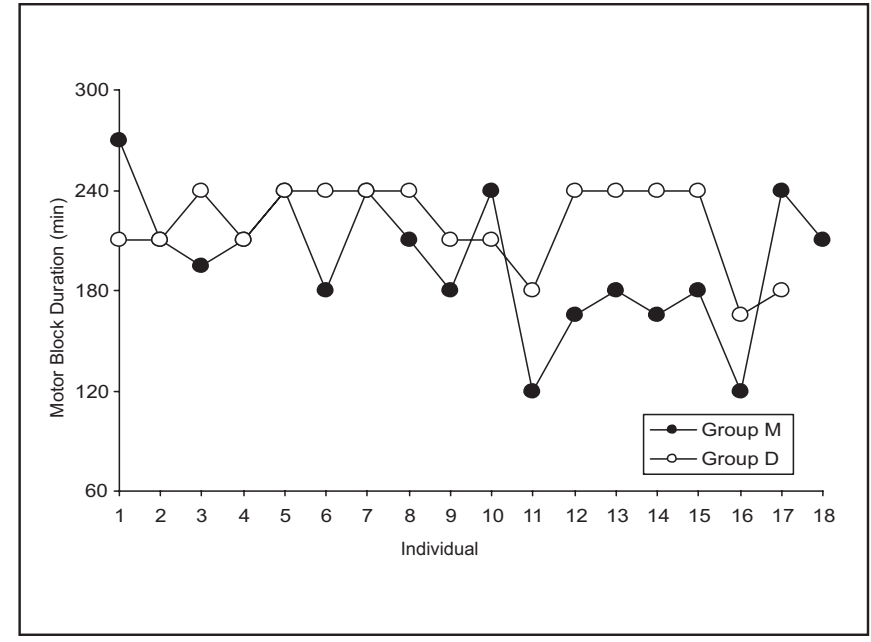

Figure 4 - Patients Distribution with Regard to Motor Block Duration

Some proposed mechanisms for the action of alpha $_{2}$-adrenergic agonists combined to local anesthetics include vasoconstriction, facilitation of $\mathrm{C}$ fibers blockade by local anesthetics, interference on retrograde axonal transportation at medullary level and action on peripheral nerve receptors ${ }^{9}$. Some reports discuss longer sensory and motor block duration induced by alpha ${ }_{2}$-adrenergic agonists during neuraxial blocks ${ }^{9}$.

Rhee et al. have observed increased sensory and motor block duration induced by intravenous clonidine associated to spinal anesthesia ${ }^{10}$. Memis et al. have observed faster motor block installation and slower recovery of total motor activity when dexmedetomidine was associated to intravenous regional anesthesia with lidocaine ${ }^{11}$. The interference of alpha $_{2}$-adrenergic agents on motor activity was not the objective of the above-mentioned studies. Studies aiming at determining the actual interference of such drug on motor and sensory block duration are needed and should be reproduced. Single or repeated dose in anesthesia could be an inadequate alternative to measure the effects of a drug. Hu et al., using mathematical simulations, have forecasted twice the variation coefficient in plasma concentration of a drug in bolus as compared to continuous infusion ${ }^{12}$. This may promote high plasma concentrations of drugs and the presence of adverse effects. Continuous infusion allows the maintenance of constant plasma concentration of drugs, preventing decreased effects and the possibility of adverse reactions. Systemic single dose as opposed to continuous infusion may be responsible for unexpected motor block duration increase. Sudo et al. have found this characteristic in an animal study where high systemic dexmedetomidine doses have increased motor block duration in animals submitted to spinal anesthesia with levobupivacaine, which was partially decreased by ioimbine ${ }^{13}$. Our study has not shown differences between dexmedetomidine or midazolam continuous infusion, in doses limited to promote sedation, with regard to sen- 
sory and motor block duration promoted by spinal anesthesia with hyperbaric $0.5 \%$ bupivacaine.

Dexmedetomidine for sedation has not changed blood pressure and heart rate values as compared to midazolam. It is possible that preanesthetic diazepam, a long half-life benzodiazepine may have influenced the level of perioperative sedation. However, both groups received the same preanesthetic medication and midazolam and dexmedetomidine infusion rates were adjusted for a target BIS value. So, we believe that decreased anesthetic need promoted by diazepam was similar for both groups. Our conclusion was that intravenous dexmedetomidine for sedation of patients submitted to spinal anesthesia is a good option for sedation, with hemodynamic stability, adequate hypnosis and without changing motor and sensory block duration.

\section{REFERÊNCIAS - REFERENCES}

01. Bernards CM - Epidural and Spinal Anesthesia, em: Barash PG, Cullen BF, Stoelting RK - Clinical Anesthesia, Philadelphia, Lippincott Williams \& Wilkins, 2001;689-713.

02. Aantaa R, Kallio A, Virtanen R - Dexmedetomidine, a novel $\alpha_{2}$-adrenergic agonist. A review of its pharmacodynamic characteristics. Drugs of the Future, 1993;18:49-56.

03. Erkola O, Korttila K, Aho M et al - Comparison of intramuscular dexmedetomidine and midazolam premedication for elective abdominal hysterectomy. Anesth Analg, 1994;79:646-653.

04. Hall JE, Uhrich TD, Barney JA et al - Sedative, amnestic, and analgesic properties of small-dose dexmedetomidine infusions. Anesth Analg, 2000;90:699-705.

05. Magalhães E, Goveia CS, Ladeira LCA et al - Relação entre a infusão contínua de dexmedetomidina e a fração expirada de sevoflurano monitorizada pelo índice bispectral. Rev Bras Anestesiol, 2004;54:303-310.

06. Aantaa R, Scheinin M - Alpha2-adrenergic agents in anaesthesia. Acta Anaesthesiol Scand, 1993;37:433-48.

07. Hayashi Y, Maze M - Alpha2-adrenoceptor agonists and anaesthesia. Br J Anaesth, 1993;71:108-118.

08. Alves TCA, Braz JRC, Vianna PTG - Alfa2-agonistas em Anestesiologia: aspectos clínicos e farmacológicos. Rev Bras Anestesiol, 2000;50:396-404.

09. Gabriel JS, Gordin V - Alpha 2 agonists in regional anesthesia and analgesia. Curr Opin Anaesthesiol, 2001;14:751-753.

10. Rhee K, Kang K, Kim J et al - Intravenous clonidine prolongs bupivacaine spinal anesthesia. Acta Anaesthesiol Scand, 2003;47:1001-1005
11. Memis D, Turan A, Karamanlioglu B et al - Adding dexmedetomidine to lidocaine for intravenous regional anesthesia. Anesth Analg, 2004;98:835-40.

12. Hu C, Horstman DJ, Shafer SL - Variability of target-controlled infusion is less than the variability after bolus injection. Anesthesiology, 2005;102:639-645.

13. Sudo RT, Calasans-Maia JA, Zapata-Sudo G - Systemic administration of dexmedetomidine increased the duration of spinal anesthesia induced by levobupivacaine. Anesthesiology, 2003;99:A955.

\section{RESUMEN}

Magalhães E, Ladeira LCA, Govêia CS, Espíndola BV - La Dexmedetomidina para Sedación, por Vía Venosa, no Interfiere con la Duración del Bloqueo Sensitivo y Motor de la Raquianestesia

JUSTIFICATIVA Y OBJETIVOS: La anestesia regional se practica con frecuencia en anestesiología. No obstante, algunos pacientes pueden mostrar ansiedad, necesitando en ese caso sedación. Los benzodiazepínicos, opioides y propofol son ampliamente utilizados para este propósito. Los agonistas alfa 2-adrenérgicos tienen propiedades hipnóticas y sedativas y son una alternativa terapéutica por la estabilidad hemodinámica y mínima depresión respiratoria. El objetivo de este estudio es evaluar la seguridad e interacciones de dexmedetomidina y midazolam por vía intravenosa en la duración del bloqueo sensitivo y motor de la raquianestesia.

MÉTODO: Fueron estudiadas 35 pacientes adultas, estado físico ASA I y Il, que recibieron raquianestesia con bupivacaína a 0,5\% hiperbárica (15 mg), para cirugía ginecológica electiva, distribuidas de modo aleatorio en 2 grupos: grupo $M(n=17)$ sedación con midazolam en infusión continua a 0,25 $\mu \mathrm{g} \cdot \mathrm{kg}^{-1} \cdot \mathrm{min}^{-1}$ y grupo $D(n=18)$ - sedación con dexmedetomidina en infusión continua a $0,5 \mu \mathrm{g} \cdot \mathrm{kg}^{-1} \cdot \mathrm{min}^{-1}$. La velocidad de infusión fue ajustada para mantener el valor del $B I S$ entre 60 y 80. Fueron analizados los valores de PAS, PAD, $\mathrm{FC}, \mathrm{SpO}_{2}$, BIS, extensión y duración del bloqueo sensitivo motor (escala de Bromage).

RESULTADOS: No hubo diferencia estadística entre los grupos en edad, peso, nivel de bloqueo sensitivo y duración bloqueo sensitivo motor, como de la frecuencia cardíaca y presión arterial.

CONCLUSIONES: La dexmedetomidina utilizada en sedación, por vía venosa, no alteró los parámetros hemodinámicos, duración o extensión de los bloqueos sensitivo y motor de la raquianestesia, representando una buena opción para sedación durante anestesia regional. 